Document downloaded from:

http://hdl.handle.net/10251/149725

This paper must be cited as:

Bonet Solves, JA.; Domanski, P. (2017). A Note on the Spectrum of Composition Operators on Spaces of Real Analytic Functions. Complex Analysis and Operator Theory. 11(1):161174. https://doi.org/10.1007/s11785-016-0589-5

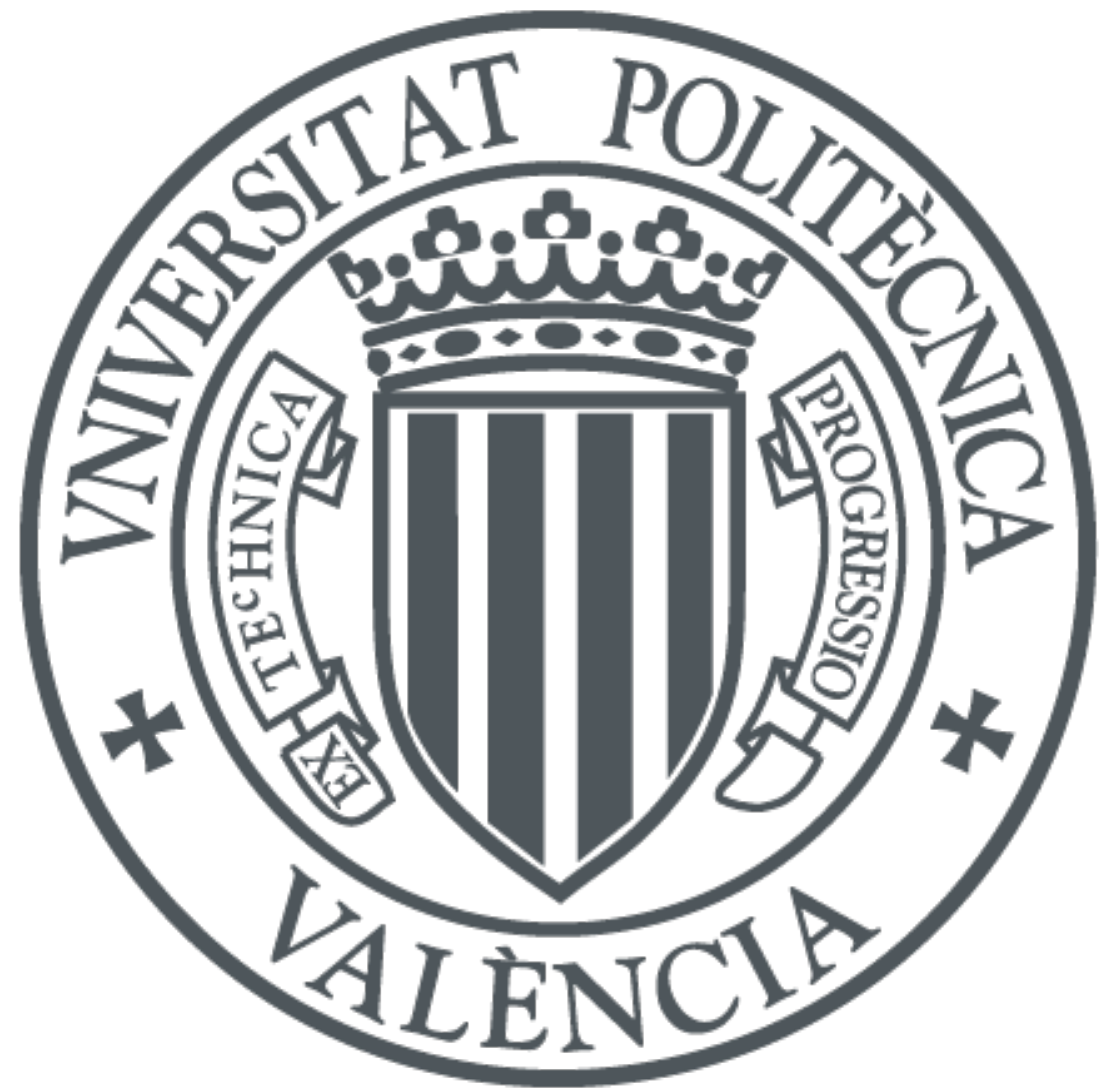

The final publication is available at

https://doi.org/10.1007/s11785-016-0589-5

Copyright Springer-Verlag

Additional Information 


\title{
A NOTE ON THE SPECTRUM OF COMPOSITION OPERATORS ON SPACES OF REAL ANALYTIC FUNCTIONS
}

\author{
José Bonet And PaWee Domański
}

\author{
Authors' Addresses: \\ J. Bonet (corresponding author) \\ Instituto Universitario de Matemática Pura \\ y Aplicada IUMPA \\ Universitat Politècnica de València \\ E-46071 Valencia, SPAIN \\ e-mail: jbonet@mat.upv.es \\ phone: +34963879497 \\ fax: +34963879497
}

P. Domański

Faculty of Mathematics and Comp. Sci.

A. Mickiewicz University Poznań

Umultowska 87

61-614 Poznań, POLAND

e-mail: domanski@amu.edu.pl

\begin{abstract}
In this paper the spectrum of composition operators on the space of real analytic functions is investigated. In some cases it is completely determined while in some other cases it is only estimated.
\end{abstract}

\footnotetext{
${ }^{1} 2010$ Mathematics Subject Classification. Primary: 47B33, 46E10. Secondary: 47A10.

Key words and phrases: Spaces of real analytic functions, composition operator, spectrum.
} 


\section{Introduction}

Let $\varphi: \mathbb{R} \rightarrow \mathbb{R}$ be a non-constant real analytic map and let $\mathscr{A}(\mathbb{R})$ be the space of real analytic functions defined on $\mathbb{R}$. Each symbol $\varphi: \mathbb{R} \rightarrow \mathbb{R}$ defines a composition operator $C_{\varphi}: \mathscr{A}(\mathbb{R}) \rightarrow$ $\mathscr{A}(\mathbb{R})$ by $C_{\varphi}(f):=f \circ \varphi, f \in \mathscr{A}(\mathbb{R})$. When $\mathscr{A}(\mathbb{R})$ is endowed with its natural locally convex topology (see e.g. [9]), $C_{\varphi}$ is a continuous linear operator on $\mathscr{A}(\mathbb{R})$. In our article [7] we studied the eigenvalues and eigenvectors of composition operators $C_{\varphi}: \mathscr{A}(\mathbb{R}) \rightarrow \mathscr{A}(\mathbb{R})$. In this note we complement those results with some examples and remarks concerning the spectrum of $C_{\varphi}: \mathscr{A}(\mathbb{R}) \rightarrow \mathscr{A}(\mathbb{R})$.

Our results in [7] give precise information about the injectivity of the operator $C_{\varphi}-\lambda I$ : $\mathscr{A}(\mathbb{R}) \rightarrow \mathscr{A}(\mathbb{R}), \lambda \in \mathbb{C} \backslash\{0\}$. In order to investigate the spectrum $\sigma\left(C_{\varphi}\right)$, we have to study when the operator $C_{\varphi}-\lambda I: \mathscr{A}(\mathbb{R}) \rightarrow \mathscr{A}(\mathbb{R})$ is surjective. This operator is surjective if and only if the operator $T_{\lambda}: \mathscr{A}(\mathbb{R}) \rightarrow \mathscr{A}(\mathbb{R})$, defined by $T_{\lambda} f(x):=f(x)-(1 / \lambda) f(\varphi(x)), x \in \mathbb{R}, f \in \mathscr{A}(\mathbb{R})$, is surjective. If we set $g(x, y):=(1 / \lambda) y+\gamma(x)$, for $\gamma \in \mathscr{A}(\mathbb{R})$, then $T_{\lambda}: \mathscr{A}(\mathbb{R}) \rightarrow \mathscr{A}(\mathbb{R})$ is surjective if and only if for each $\gamma \in \mathscr{A}(\mathbb{R})$ there is $f \in \mathscr{A}(\mathbb{R})$ such that $f(x)=g(x, f(\varphi(x)))$, $x \in \mathbb{R}$. We use methods of the paper [4].

Equations of the form $f(x)=g\left(x, f(\varphi(x))\right.$ with $g: \mathbb{R}^{2} \rightarrow \mathbb{R}$ and $\varphi: \mathbb{R} \rightarrow \mathbb{R}$ real analytic are considered in [4] and [17] (see also [1], [2] or the book [3]). As it is mentioned in the introduction of [4], if $u$ is a fixed point of $\varphi$ and $\lambda \neq \varphi^{\prime}(u)^{n}$ for each $n \in \mathbb{N}_{0}$, then $f(x)=(1 / \lambda) f(\varphi(x))+\gamma(x)$ has a formal solution $f(x)=u+\sum_{n=1}^{\infty} c_{n}(x-u)^{n}$. Smajdor [17] studies conditions to ensure that this series representation has a positive radius of convergence near $u$.

In the rest of the article we denote id $(x)=x, x \in \mathbb{R}$, and, for a map $\varphi: \mathbb{R} \rightarrow \mathbb{R}$, we write $\varphi^{[0]}=$ id and $\varphi^{[n]}$ for the $n$-times composition of $\varphi, n \in \mathbb{N}$. By $I: \mathscr{A}(J) \rightarrow \mathscr{A}(J)$ we denote the identity operator.

The composition operators on spaces of real analytic functions have been considered in several papers, like [9], [10], [11], [12], [13], [5], [6]. For literature on the space of real analytic functions see a recent survey [9]. For functional analytic tools see [16].

\section{Preliminaries}

By interval $J$ we mean also unbounded ones (i.e., halflines or the whole real line). It is clear that results on the (point) spectrum of a composition operator on $\mathscr{A}(\mathbb{R})$ can be easily transferred to the case of $\mathscr{A}(J)$ where $J$ is an open interval in $\mathbb{R}$. We recall the following two results from [7].

Proposition 2.1 Let $\varphi: J \rightarrow J$ be a real analytic function on an open interval $J \subset \mathbb{R}$ and let $C_{\varphi}: \mathscr{A}(J) \rightarrow \mathscr{A}(J)$ be the associated composition operator. Then

(1) ([7, Proposition $1.1(4)]) 0 \in \sigma\left(C_{\varphi}\right)$ if and only if $\varphi: J \rightarrow J$ is not a real analytic diffeomorphism.

(2) ([7, Proposition 2.8 (4)]) If $u \in J$ is a fixed point of $\varphi$ such that $\left|\varphi^{\prime}(u)\right| \neq 0,1$, then $\varphi^{\prime}(u)^{n} \in \sigma\left(C_{\varphi}\right)$ for each $n \in \mathbb{N}_{0}$.

We say that some subset $A$ of an open interval $J \subset \mathbb{R}$ is bounded away from the upper end of $J$ if there is $\delta \in J$ such that all elements of $A$ are $<\delta$. Analogously we define subsets bounded away form the lower end of $J$. We also use the following description of the point spectrum of $C_{\varphi}$ from [7]:

Theorem 2.2 Let $\varphi: J \rightarrow J$ be a real analytic map for some open interval $J \subset \mathbb{R}$. 
(a) If $\varphi$ has no fixed points and the set of its critical points is bounded away from the upper end of $J$ (in case $\varphi>\mathrm{id}$ ) or from the lower end of $J$ (in case $\varphi<\mathrm{id}$ ) then $\sigma_{p}\left(C_{\varphi}\right)=\mathbb{C} \backslash\{0\}$ and every eigenspace is isomorphic to $\mathscr{A}(\mathbb{T})$.

(b) If $\varphi$ has a fixed point $u$ then:

(b1) if $\varphi^{[2]}$ has exactly one fixed point and either $0<\left|\varphi^{\prime}(u)\right|<1$ or $1<\left|\varphi^{\prime}(u)\right|$ and $\varphi$ has no critical points then

$$
\sigma_{p}\left(C_{\varphi}\right)=\left\{\left(\varphi^{\prime}(u)\right)^{n}: n=0,1, \ldots\right\}
$$

and the eigenspaces are all one dimensional;

(b2) if $\varphi^{[2]}=\mathrm{id} \neq \varphi$ then $\sigma_{p}\left(C_{\varphi}\right)=\{-1,1\}$ and the eigenspaces are isomorphic to $\mathscr{A}_{+}(\mathbb{R})$ the space of even real analytic functions;

(b3) if $\varphi=$ id then $\sigma_{p}\left(C_{\varphi}\right)=\{1\}$ and the eigenspace is equal to $\mathscr{A}(J)$.

(c) In all other cases $\sigma_{p}\left(C_{\varphi}\right)=\{1\}$ and the eigenspace consists of constant functions only.

We will use also the following result of Smajdor [17, Theorem p. 40]:

Theorem 2.3 Let $\varphi$ be a holomorphic function of one complex variable on a neighbourhood of $u \in \mathbb{C}, \varphi(u)=u,\left|\varphi^{\prime}(u)\right|<1$. Let $h$ be a holomorphic function of two complex variables on $a$ neighbourhood of $(u, v) \in \mathbb{C}^{2}, h(u, v)=v$. If there is a formal solution $f(z)=\sum_{n} f_{n}(z-u)^{n}$ of the equation

$$
f(z)=h(z, f(\varphi(z)), \quad f(u)=v
$$

then there is a holomorphic solution of (1) on some neighbourhood of $u$.

In case

$$
1-\frac{\partial h}{\partial v}(u, v) \cdot\left(\varphi^{\prime}(u)\right)^{n} \neq 0 \quad \text { for every } n=0,1, \ldots
$$

the formal solution is unique and so it is convergent around $u$ and gives a holomorphic solution of (1) on some neighbourhood of $u$.

Please note that in the proof of Proposition 4.1 we give a simple example where for $\left|\varphi^{\prime}(u)\right|=1$ the result above fails.

\section{Spectrum}

The case of $\varphi: J \rightarrow J$ without fixed points is mostly solved.

Corollary 3.1 Let $\varphi: J \rightarrow J$ be real analytic, $J \subset \mathbb{R}$ an open interval, and $\varphi$ have no fixed points.

(a) If $\varphi$ is a diffeomorphism onto $J$ then $\sigma\left(C_{\varphi}\right)=\sigma_{p}\left(C_{\varphi}\right)=\mathbb{C} \backslash\{0\}$.

(b) If $\varphi$ is not a diffeomorphism onto $J$ but the set of critical points of $\varphi$ is bounded away from the upper end of $J$ (in case $\varphi>$ id) or from lower end of $J$ (in case $\varphi<$ id) then $\sigma\left(C_{\varphi}\right)=\mathbb{C}$ and $\sigma_{p}\left(C_{\varphi}\right)=\mathbb{C} \backslash\{0\}$.

Proof: Combine Proposition 2.1 and Theorem 2.2. 
Problem 3.2 Describe $\sigma\left(C_{\varphi}\right)$ if $\varphi>$ id has no fixed points but the set of critical points of $\varphi$ is not bounded away from the upper end of the domain.

Now, we concentrate on the fixed point case. We will need the following standard extension lemma (this procedure for diffeomorphisms was used in [4], we write it precisely in the general case).

Lemma 3.3 Let $\varphi:(a, b) \rightarrow(a, b)$ be a real analytic map on an open interval $(a, b) \subset \mathbb{R}$ and let $\varphi(c, d) \subset(c, d) \subset[c, d] \subset(a, b)$ for some fixed $a, b, c, d$. Assume that for every $x \in(a, b)$ there is $n \in \mathbb{N}$ such that $\varphi^{[n]}(x) \in(c, d)$ (i.e., $(a, b)$ is the attraction basin for $(c, d)$ ).

If $\tilde{f} \in \mathscr{A}(c, d)$ satisfies

$$
\tilde{f}(\varphi(x))-\lambda \tilde{f}(x)=\gamma(x) \quad \text { for every } x \in(c, d),
$$

for some fixed $\lambda \in \mathbb{C} \backslash\{0\}$ and $\gamma \in \mathscr{A}(a, b)$, then $\tilde{f}$ extends to $f \in \mathscr{A}(a, b)$ satisfying

$$
f(\varphi(x))-\lambda f(x)=\gamma(x) \quad \text { for every } x \in(a, b) .
$$

Proof: Define

$$
J_{n}:=\left\{x \in(a, b) \mid \varphi^{[n]}(x) \in(c, d)\right\} .
$$

Clearly, $\left(J_{n}\right)$ is an increasing open exhaustion of $(a, b)$. Take any compact increasing exhaustion $\left(\left[a_{n}, b_{n}\right]\right)_{n \in \mathbb{N}}$ with

$$
(c, d) \subset\left[a_{n}, b_{n}\right] \subset(a, b) \text { and } \bigcup_{n \in \mathbb{N}}\left[a_{n}, b_{n}\right]=(a, b) .
$$

Without loss of generality we may assume that $J_{n} \supset\left[a_{n}, b_{n}\right]$.

We define inductively:

$$
f_{0}:=\tilde{f}, \quad f_{n}(x):=\frac{1}{\lambda}\left(\gamma(x)+f_{n-1}(\varphi(x))\right) \quad \text { for } x \in J_{n} .
$$

By (3) it is easy to observe that $f_{n}=\tilde{f}$ on $(c, d)$ and thus $f_{n}$ extends $\tilde{f}$ on $\left[a_{n}, b_{n}\right]$. Now, defining $f(x):=f_{n}(x)$ for $x \in\left[a_{n}, b_{n}\right]$ we obtain a real analytic function $f \in \mathscr{A}(a, b)$. Since

$$
f(\varphi(x))-\lambda f(x)=\gamma(x) \quad \text { for every } x \in(c, d)
$$

and since both sides of the above equality are analytic on $(a, b)$ so the equality holds everywhere on $(a, b)$.

Note that if $\varphi^{[2]}$ has the unique fixed point $u$ then $u$ must be the unique fixed point of $\varphi$ as well since otherwise $\varphi(u)=w \neq u$ but then $\varphi^{[2]}(w)=w$ and $\varphi^{[2]}$ would have had two fixed points $u$ and $w$.

Theorem 3.4 Let $\varphi: J \rightarrow J$ be a real analytic function on an open interval $J \subset \mathbb{R}$ and let $C_{\varphi}: \mathscr{A}(J) \rightarrow \mathscr{A}(J)$ the associated composition operator. Suppose that $\varphi^{[2]}$ has a unique fixed point $u$ and $\left|\varphi^{\prime}(u)\right|<1$. Then

(i) If $\lambda \neq 0$ and $\lambda \neq \varphi^{\prime}(u)^{n}$ for each $n \in \mathbb{N}_{0}$, then $\lambda \notin \sigma\left(C_{\varphi}\right)$, and

(ii) $\operatorname{ker}\left(C_{\varphi}-\lambda I\right)$ is finite dimensional for all $\lambda \in \mathbb{C}$.

Remark 3.5 The case of $\varphi$ diffeomorphic onto is proved in [4, Th. 4.4]. 
Proof: (i): Consider $\lambda \neq 0, \lambda \neq \varphi^{\prime}(u)^{n}, n \in \mathbb{N}_{0}$. By Theorem 2.2, $C_{\varphi}-\lambda I$ is injective, we will show that it is surjective.

It suffices to solve, for every $\gamma \in \mathscr{A}(J)$, the equation

$$
f(x)-(1 / \lambda) f(\varphi(x))=\gamma(x)
$$

which is equivalent to

$$
f(x)=g(x, f(\varphi(x)),
$$

with $g(x, y)=\gamma(x)+(1 / \lambda) y$. In order to apply Theorem 2.3 to the equation above, we define $v:=\lambda \gamma(u) /(\lambda-1)$. Clearly, $g(u, v)=v$. Since

$$
\frac{\partial g}{\partial v}(u, v)=\frac{1}{\lambda},
$$

the condition (2) is satisfied.

Therefore all the assumptions of Theorem 2.3 are satisfied and the unique formal solution $f_{0}$ is real analytic on a neighbourhood of $u$. Hence there is $r>0$ such that $[u-r, u+r] \subset J$ and $f_{0} \in \mathscr{A}(u-r, u+r)$ satisfies

$$
f_{0}(x)-1 / \lambda f(\varphi(x))=\gamma(x) \quad \text { for every } x \in(u-r, u+r) .
$$

Since $\left|\varphi^{\prime}(u)\right|<1$, we may assume without loss generality that $\left.\varphi(] u-r, u+r[) \subset\right] u-r, u+r[$. In the proof of $[7$, Theorem 2.6] it is proved that if $\varphi: J \rightarrow J, J \subset \mathbb{R}$ an open interval, has a fixed point $u,\left|\varphi^{\prime}(u)\right|<1$, and $\varphi^{[2]}$ has exactly one fixed point, then for every $x \in J$ holds $\varphi^{[n]}(x) \rightarrow u$ as $n \rightarrow \infty$. Thus $(c, d)=(u-r, u+r)$ satisfies the assumptions of Lemma 3.3. This completes the proof.

(ii): This follows from results of [7, Section 2] transferring them to an arbitrary interval instead of the whole line. For readers convenience we sketch the proof. By assumption $\varphi^{[2]}$ has a unique fixed point in $J$ and we can aply [7, Theorem 2.6] to conclude that $\varphi^{\prime}(u)^{n}$ is an eigenvalue of $C_{\varphi}$ for each $n \in \mathbb{N}_{0}$. By [7, Theorem 2.9 (c) and (d)], $\operatorname{ker}\left(C_{\varphi}-\lambda I\right)$ is one dimensional for $\lambda=0$ (if $\varphi^{\prime}(u)=0$ ) or $\lambda=\varphi^{\prime}(u)^{n}$ (if $0<\left|\varphi^{\prime}(u)\right|<1$ ). We can apply [7, Propositions 1.1 and 2.4] to conclude that $\operatorname{ker}\left(C_{\varphi}-\lambda I\right)=\{0\}$ for $\lambda \neq \varphi^{\prime}(u)^{n}, n \in \mathbb{N}_{0}$.

Corollary 3.6 If $\varphi: J \rightarrow J$ is a real analytic on an open interval $J \subset \mathbb{R}$, such that $\varphi^{[2]}$ has the unique fixed point $u$ satisfying $\left|\varphi^{\prime}(u)\right|<1$, then

$$
\sigma\left(C_{\varphi}\right)= \begin{cases}\left\{\varphi^{\prime}(u)^{n} \mid n \in \mathbb{N}_{0}\right\} & \text { if } \varphi \text { is a diffeomorphism, } \\ \left\{\varphi^{\prime}(u)^{n} \mid n \in \mathbb{N}_{0}\right\} \cup\{0\} & \text { otherwise }\end{cases}
$$

and

$$
\sigma_{p}\left(C_{\varphi}\right)=\left\{\varphi^{\prime}(u)^{n} \mid n \in \mathbb{N}_{0}\right\} \backslash\{0\}
$$

Moreover, $\operatorname{ker}\left(C_{\varphi}-\lambda I\right)$ is finite dimensional for all $\lambda \in \mathbb{C}$.

Proof: The description of $\sigma_{p}\left(C_{\varphi}\right)$ follows from Theorem 2.2. The description of $\sigma\left(C_{\varphi}\right)$ follows combining of Proposition 2.1 and Theorem 3.4.

Corollary 3.7 If $\varphi: J \rightarrow J$ is a real analytic diffeomorphism on an open interval $J \subset \mathbb{R}$ such that $\varphi^{[2]}$ has a unique fixed point $u$ satisfying $\left|\varphi^{\prime}(u)\right|>1$, then $\sigma\left(C_{\varphi}\right)=\sigma_{p}\left(C_{\varphi}\right)=\left\{\varphi^{\prime}(u)^{n} \mid n \in\right.$ $\left.\mathbb{N}_{0}\right\}$ and $\operatorname{ker}\left(C_{\varphi}-\lambda I\right)$ is finite dimensional for all $\lambda \in \mathbb{C}$. 
Proof: The map $\psi:=\varphi^{-1}: J \rightarrow J$ is a real analytic diffeomorphism with a unique fixed point $u \in J$ and $0<\left|\psi^{\prime}(u)\right|<1$. The conclusion follows from Corollary 3.6 and the following observation: for $\mu \neq 0, \mu \neq \psi^{\prime}(u)^{n}=1 / \varphi^{\prime}(u)^{n}, n \in \mathbb{N}_{0}$, the operator $C_{\psi}-\mu I: \mathscr{A}(J) \rightarrow \mathscr{A}(J)$ is surjective/injective if and only if $C_{\varphi}-(1 / \mu) I: \mathscr{A}(J) \rightarrow \mathscr{A}(J)$ is surjective/injective.

Problem 3.8 Describe $\sigma\left(C_{\varphi}\right)$ if $\varphi$ has a fixed point $u,\left|\varphi^{\prime}(u)\right|>1, \varphi^{[2]}$ has a unique fixed point but $\varphi$ is not a diffeomorphism.

By $[4$, Theorem 4.5] we obtain immediately:

Proposition 3.9 Let $\varphi: j \rightarrow J, J \subset \mathbb{R}$ open interval, be a real analytic diffeomorphism with fixed points $x_{1}<x_{2}<\ldots<x_{s}$ such that $\left|\varphi^{\prime}\left(x_{i}\right)\right| \neq 1$ for $i=1,2, \ldots, s, s>1$. For each $\lambda \neq 0$, the operator $C_{\varphi}-\lambda I: \mathscr{A}(J) \rightarrow \mathscr{A}(J)$ has closed range, $\operatorname{ker} C_{\varphi}-\lambda I=\{0\}$ if $\lambda \neq 1, \operatorname{ker} C_{\varphi}-I$ is finite dimensional and the codimension of $\operatorname{im} C_{\varphi}-\lambda I$ in $\mathscr{A}(J)$ is infinite. In particular, $\sigma_{p}\left(C_{\varphi}\right)=\{1\}$ and $\sigma\left(C_{\varphi}\right)=\mathbb{C} \backslash\{0\}$.

Proof: By Proposition $2.1(1), 0 \notin \sigma\left(C_{\varphi}\right)$ and by Theorem 2.2, $\sigma_{p}\left(C_{\varphi}\right)=\{1\}$, and $\operatorname{ker}\left(C_{\varphi}-\right.$ $I)$ is one dimensional. The rest of the statement is a direct consequence of [4, Theorem 4.5].

Example 3.10 (a) If $\varphi(x)=e^{\alpha x}, \alpha>1 / e$, then $\sigma_{p}\left(C_{\varphi}\right)=\mathbb{C} \backslash\{0\}$ and $\sigma\left(C_{\varphi}\right)=\mathbb{C}$ (use Corollary $3.1)$.

(b) If $\varphi$ has no fixed points on $\mathbb{R}$ and $\varphi$ has only finitely many critical points, then $\sigma_{p}\left(C_{\varphi}\right)=$ $\mathbb{C} \backslash\{0\}$ and $\sigma\left(C_{\varphi}\right)=\mathbb{C}$ by Corollary 3.1.

(c) If $\varphi(x)=\alpha \arctan (x), 0<\alpha<1$, then $\sigma_{p}\left(C_{\varphi}\right)=\left\{\alpha^{n} \mid n \in \mathbb{N}_{0}\right\}$ and $\sigma\left(C_{\varphi}\right)=\sigma_{p}\left(C_{\varphi}\right) \cup\{0\}$. In this case $\varphi$ has a unique fixed point $u=0$ and $\varphi^{\prime}(u)=\alpha$. We have $0<\left|\varphi^{\prime}(u)\right|<1$ and $\varphi$ is not surjective, hence the statement follows from Corollary 3.6.

Before we deal with more examples, we present for the reader's convenience the main result in [4, Section 3] in a way suitable for our purposes explaining details omitted in [4].

Let $\varphi: \mathbb{R} \rightarrow \mathbb{R}$ be a real analytic self map, let $\lambda \in \mathbb{C}, \lambda \neq 0$, and consider the operator $T: \mathscr{A}(\mathbb{R}) \rightarrow \mathscr{A}(\mathbb{R}), T f(x):=f(\varphi(x))-\lambda f(x)$. Let $\left(U_{j}\right)_{j}$ be an open covering of $\mathbb{R}$ such that $\varphi\left(U_{j}\right) \subset U_{j}$ for each $j$ and such that each $U_{j}$ intersects only finitely many other $U_{k}$ 's. Denote by $T_{k}: \mathscr{A}\left(U_{k}\right) \rightarrow \mathscr{A}\left(U_{k}\right)$ and $T_{k, j}: \mathscr{A}\left(U_{k} \cap U_{j}\right) \rightarrow \mathscr{A}\left(U_{k} \cap U_{j}\right)$ the operator $T$ defined on the corresponding space. With the notation $g \mid V$ for the restriction of the function $g$ to the set $V$, define

$$
\begin{aligned}
\mathscr{A}\left(\mathbb{R},\left(U_{k}\right), T\right) & :=\left\{\gamma \in \mathscr{A}(\mathbb{R}): \gamma \mid U_{k} \in \operatorname{im}\left(T_{k}\right) \quad \forall k\right\}, \\
E & :=\left\{\left(f_{k, j}\right)_{k, j}: f_{k, j}=-f_{j, k}, \quad f_{k, j} \in \operatorname{ker}\left(T_{k, j}\right)\right\}, \\
F & :=\left\{\left(f_{k}\right)_{k}: f_{k} \in \operatorname{ker}\left(T_{k}\right) \forall k\right\},
\end{aligned}
$$

and

$$
S: F \rightarrow E, \quad S\left(\left(f_{k}\right)_{k}:=\left(\left(f_{k}-f_{j}\right) \mid U_{k} \cap U_{j}\right)_{k, j} .\right.
$$

Theorem 3.11 ([4, Theorem 3.1]) There is a linear surjective map

$$
\mathcal{F}: \mathscr{A}\left(\mathbb{R},\left(U_{k}\right), T\right) \rightarrow E / S(F)
$$

with kernel equal to im $(T)$.

In particular, if $S(F) \neq E$, then $T$ is not surjective. 
Proof: Given $\gamma \in \mathscr{A}\left(\mathbb{R},\left(U_{k}\right), T\right)$, for each $k$ there is $f_{k} \in \mathscr{A}\left(U_{k}\right)$ such that $T_{k} f_{k}=\gamma \mid U_{k}$. Set $f_{k, j}:=\left(f_{k}-f_{j}\right) \mid U_{k} \cap U_{j}$. Clearly $T_{k, j} f_{k, j}(x)=0$ for each $x \in U_{k} \cap U_{j}$ and each $k, j$. Hence $\left(f_{k, j}\right)_{k, j} \in E$. We define $\mathcal{F}(\gamma)=\left(f_{k, j}\right)_{k, j}+S(F)$. To see that $\mathcal{F}$ is well defined, suppose that $T_{k} g_{k}=\gamma \mid U_{k}$ with $g_{k} \in \mathscr{A}\left(U_{k}\right)$ and set $g_{k, j}:=\left(g_{k}-g_{j}\right) \mid U_{k} \cap U_{j}$. We have $\left(f_{k, j}-g_{k, j}\right)_{k, j}=$ $S\left(\left(f_{k}-g_{k}\right)_{k}\right)$ and $T_{k}\left(f_{k}-g_{k}\right)=0$ for each $k$. Therefore $\left(f_{k, j}\right)_{k, j}-\left(g_{k, j}\right)_{k, j} \in S(F)$ and $\mathcal{F}$ is indeed well defined.

We show now that $\mathcal{F}$ is surjective. Fix $\left(f_{k, j}\right)_{k, j}+S(F) \in E / S(F)$. Our assumptions on the covering $\left(U_{k}\right)_{k}$ of $\mathbb{R}$ permit us to find open sets $V_{k}$ in the complex plane with $V_{k} \cap \mathbb{R}=U_{k}$ and extensions of the functions $f_{k, j}$ to holomorphic functions $g_{k, j}$ on $V_{k} \cap V_{j}$.

Indeed, let $f_{k, j} \in \mathscr{A}\left(U_{k} \cap U_{j}\right)$ then there is an open complex neighbourhood $W$ of $U_{k} \cap U_{j}$, $W \cap \mathbb{R}=U_{k} \cap U_{j}$, such that $f_{k, j}$ extends holomorphically as a function $g_{k, j}$ on $W$. We define:

$$
\tau_{j}(x):= \begin{cases}\min \left(d\left(x, \partial_{\mathbb{R}} U_{j}\right), d\left(x, \partial_{\mathbb{C}} W\right)\right) & \text { if } x \in U_{k} \cap U_{j} \text { and } d\left(x, \partial_{\mathbb{R}} U_{j}\right) \leq d\left(x, \partial_{\mathbb{R}} U_{k}\right), k \neq j ; \\ d\left(x, \partial_{\mathbb{R}} U_{j}\right) & \text { otherwise. }\end{cases}
$$

We define $\tau_{k}$ analogously. Then we define open sets $V_{j}$ and $V_{k}$, for instance,

$$
V_{j}:=\left\{x+i y \in \mathbb{C}^{d}: x \in U_{j},|y|<\tau_{j}(x)\right\} .
$$

Then

$$
V_{k} \cap V_{j} \subset W, \quad V_{k} \cap V_{j} \cap \mathbb{R}=U_{k} \cap U_{j}, \quad V_{j} \cap \mathbb{R}=U_{j}, \quad V_{k} \cap \mathbb{R}=U_{k} .
$$

If $U_{k}$ intersects more sets $U_{j}$ (but finitely many) then we can take intersections of the obtained sets $V_{k}$. In that way we get $g_{k, j}$ holomorphic on $V_{k} \cap V_{j}$.

Since for the orthogonal projection $p: \mathbb{C}=\mathbb{R}^{2} \rightarrow \mathbb{R}$ we have $p\left(V_{j}\right)=V_{j} \cap \mathbb{R}=U_{j}$ (see the definition of $V_{j}$ ), thus

$$
p\left(V_{j} \cap V_{k} \cap V_{l}\right)=U_{j} \cap U_{k} \cap U_{l}
$$

and every connected component of $V_{j} \cap V_{k} \cap V_{l}$ contains a non-empty open part of $U_{j} \cap U_{k} \cap U_{l}$. As easily seen on the intersection $U_{j} \cap U_{k} \cap U_{l}$ it holds $f_{j, k}+f_{k, l}+f_{l, j}=0$ by the very definition of the functions. Thus also extensions satisfy $g_{j k}+g_{k l}+g_{l j}=0$ on $V_{j} \cap V_{k} \cap V_{l}$ for any triple $\{j, k, l\}$.

By the Cartan-Grauert result (see [8, Prop. 1]) every open set in $\mathbb{R}^{d}$ has a basis of complex neighbourhoods being domains of holomorphy. So there is a domain of holomorphy $V \subset \bigcup V_{k}$, $V \cap J=J$. Thus we can apply the solution to the first Cousin problem [15, Th. 5.5.1] for the covering $\left(V_{k} \cap V\right)$ of $V$ and Cousin data $\left(g_{j, k}\right)$ to find, after taking restrictions, $\left(f_{k}\right)_{k}$ with $f_{k} \in \mathcal{A}\left(U_{k}\right)$ such that $f_{k, j}=f_{k}-f_{j}$ for each $k, j$. Define $\gamma_{k}:=T_{k} f_{k} \in \mathcal{A}\left(U_{k}\right)$. Since $f_{k, j} \in \operatorname{ker}\left(T_{k, j}\right), \gamma_{k}(x)=\gamma_{j}(x)$ for each $x \in U_{k} \cap U_{j}$, and we can find $\gamma \in \mathscr{A}(\mathbb{R})$ such that $\gamma \mid U_{k}=\gamma_{k}=T_{k} f_{k}$ for each $k$. This means $\gamma \in \mathscr{A}\left(\mathbb{R},\left(U_{k}\right), T\right)$. Moreover $\mathcal{F}(\gamma)=\left(f_{k, j}\right)_{k, j}+S(F)$.

It remains to show that $\operatorname{ker}(\mathcal{F})=\operatorname{im}(T)$. If $\gamma \in \operatorname{im}(T)$, there is $f \in \mathscr{A}(\mathbb{R})$ such that $T f=\gamma$. Then $\gamma \in \mathscr{A}\left(\mathbb{R},\left(U_{k}\right), T\right)$ since $\gamma \mid U_{k}=T_{k}\left(f \mid U_{k}\right)$ for each $k$. Moreover, $\mathcal{F}(\gamma)=0 \in E / S(F)$ and $\gamma \in \operatorname{ker}(\mathcal{F})$.

To prove the reverse inclusion, take $\gamma \in \mathscr{A}\left(\mathbb{R},\left(U_{k}\right), T\right)$ with $\mathcal{F}(\gamma)=0 \in E / S(F)$. For each $k$ there is $f_{k} \in \mathscr{A}\left(U_{k}\right)$ such that $T_{k} f_{k}=\gamma \mid U_{k}$. By assumption $\left(\left(f_{k}-f_{j}\right) \mid U_{k} \cap U_{j}\right)_{k, j} \in S(F)$. Therefore we can find $\left(g_{k}\right)_{k}, g_{k} \in \mathscr{A}\left(U_{k}\right), T_{k} g_{k}=0$ such that $f_{k}-f_{j}=g_{k}-g_{j}$ on $U_{k} \cap U_{j}$ for each $k, j$. Hence $f_{k}-g_{k}=f_{j}-g_{j}$ on $U_{k} \cap U_{j}$ for each $k, j$. Accordingly, there is $f \in \mathscr{A}(\mathbb{R})$ such that $f \mid U_{k}=f_{k}-g_{k}$ for each $k$. If $x \in U_{k}$, we get $T f(x)=T_{k} f_{k}(x)-T_{k} g_{k}(x)=\gamma(x)$. Since $\left(U_{k}\right)$ is an open covering of $\mathbb{R}$ we get $T f=\gamma$ and $\gamma \in \operatorname{im}(T)$.

Proposition 3.12 If $\varphi(x)=x^{s}, s \in \mathbb{N}, s>1$, then for $C_{\varphi}: \mathscr{A}(\mathbb{R}) \rightarrow \mathscr{A}(\mathbb{R})$ we have that $\sigma_{p}\left(C_{\varphi}\right)=\{1\}, \operatorname{ker}\left(C_{\varphi}-I\right)$ is one dimensional and its elements are the constants, but $\sigma\left(C_{\varphi}\right)=\mathbb{C}$. 
Proof: The statement on eigenvalues and eigenspaces follows by Theorem 2.2.

Now, we consider the spectrum. First of all, since $\varphi$ is not a diffeomorphism, $0 \in \sigma\left(C_{\varphi}\right)$ by Proposition 2.1. The map $\varphi$ has three fixed points $0,1,-1$ if $s$ is odd and two 0,1 if $s$ is even. Anyway, $\varphi^{\prime}(1)=s$ (which coincides with $\varphi^{\prime}(-1)$ if $s$ is odd). By Proposition 2.1, as $s \neq 1$, $s^{n} \in \sigma\left(C_{\varphi}\right)$ for each $n \in \mathbb{N}_{0}$. It remains to show that

$$
T:=C_{\varphi}-\lambda I: \mathscr{A}(\mathbb{R}) \rightarrow \mathscr{A}(\mathbb{R})
$$

is not surjective for $\lambda \neq 0, \lambda \neq s^{n}, n \in \mathbb{N}_{0}$.

We consider first the case when $s$ is odd.

Set $U_{-1}:=(-\infty, 0), U_{0}:=(-1,1), U_{1}:=(0, \infty)$, that are invariant sets under $\varphi$ and they cover $\mathbb{R}$. Denote by $T_{k}$ the restriction of $T=C_{\varphi}-\lambda I$ to $\mathscr{A}\left(U_{k}\right), k=-1,0,1$. By Theorem 3.4, $\operatorname{ker} T_{0}$ is finite dimensional, since $\varphi$ and $\varphi^{[2]}$ have on $U_{0}$ only one fixed point. On the other hand, since $\varphi$ is a real analytic diffeomorphism both in $U_{-1}$ and in $U_{1}$, we can apply Corollary 3.7 to conclude that $\operatorname{ker} T_{i}$ is finite dimensional for $i=-1,1$. Now set $U_{-1,0}:=U_{-1} \cap U_{0}=(-1,0)$ and $U_{0,1}:=U_{0} \cap U_{1}=(0,1)$ and $T_{i, k}$ for the operator $T$ restricted to $\mathcal{A}\left(U_{i, k}\right)$. Each $U_{i, k}$ is invariant under $\varphi$ and $\varphi$ is a real analytic diffeomorphism on each $U_{i, k}$ without fixed points. We can apply Theorem 2.2 to obtain that each $\operatorname{ker} T_{i, k}$ is infinite dimensional.

In the notation of Theorem 3.11, we have that $S: F \rightarrow E$ is not surjective, because the domain is finite dimensional and the range is infinite dimensional. We can apply Theorem 3.11 to conclude that $C_{\varphi}-\lambda I: \mathscr{A}(\mathbb{R}) \rightarrow \mathscr{A}(\mathbb{R})$ is not surjective (even more is true: codim im $C_{\varphi}-\lambda I=$ $\infty)$.

Now suppose that $s$ is even. We define $U_{0}=\mathbb{R} \backslash\{0\}, U_{1}=(-1,1)$. They are invariant with respect to $\varphi$. We will describe $\left.\operatorname{ker} T\right|_{\mathscr{A}\left(U_{i}\right)}$.

First, if $\left.f \in \operatorname{ker} T\right|_{\mathscr{A}\left(U_{0}\right)}$, then for $x<0$ we can calculate $f(x)=(1 / \lambda) f\left(x^{S}\right)$ so it is uniquely determined by $\left.f\right|_{\mathbb{R}_{+}}$and the latter function belongs to $\left.\operatorname{ker} T\right|_{\mathscr{A}\left(\mathbb{R}_{+}\right)}$. Since $\varphi$ is a diffeomorphism and $\varphi^{[2]}$ has one fixed point on $\mathbb{R}_{+}$thus by Corollary 3.7, the kernel is finite dimensional. Now, $\left.\operatorname{ker} T\right|_{\mathscr{A}\left(U_{1}\right)}$ is finite dimensional by Theorem 3.4.

For the space $\mathscr{A}\left(U_{0} \cap U_{1}\right)$ the kernel of $T_{\lambda}$ is infinite dimensional, since again its elements $f$ are determined uniquely by $\left.f\right|_{(0,1)}$ and the latter belongs to $\left.\operatorname{ker} T\right|_{\mathscr{A}(0,1)}$. By Theorem 2.2, this kernel is infinite dimensional.

We apply again Theorem 3.11 to conclude that $T=C_{\varphi}-\lambda I: \mathscr{A}(\mathbb{R}) \rightarrow \mathscr{A}(\mathbb{R})$ is not surjective, since the map $S: F \rightarrow E$ is not surjective. Indeed, the domain is finite dimensional but the range space is infinite dimensional and so codimim $C_{\varphi}-\lambda I=\infty$.

In order to get more examples, the following observation is useful. The proof is easy.

Lemma 3.13 Let $\varphi: \mathbb{R} \rightarrow \mathbb{R}$ be a real analytic self map and let $\delta: \mathbb{R} \rightarrow \mathbb{R}$ be a real analytic diffeomorphism. The real analytic self map $\psi: \mathbb{R} \rightarrow \mathbb{R}, \psi:=\delta^{-1} \circ \varphi \circ \delta$, satisfies $\sigma_{p}\left(C_{\psi}\right)=\sigma_{p}\left(C_{\varphi}\right)$ and $\sigma\left(C_{\psi}\right)=\sigma\left(C_{\varphi}\right)$. Moreover, $u$ is a fixed point of $\varphi$ if and only if $\delta^{-1}(u)$ is a fixed point of $\psi$. If $u$ is a fixed point of $\varphi$, then $\psi^{\prime}\left(\delta^{-1}(u)\right)=\varphi^{\prime}(u)$. Moreover, a subset $A$ of $\mathbb{R}$ is invariant by $\varphi$ if and only if $\delta^{-1}(A)$ is invariant for $\psi$.

As an immediate consequence of Lemma 3.13 and Example 3.12, we get

Example 3.14 Let $\delta: \mathbb{R} \rightarrow \mathbb{R}$ be a real analytic diffeomorphism and let $s \in \mathbb{N}, s>1$. The real analytic self map $\varphi(x):=\delta^{-1}\left(\delta(x)^{s}\right), x \in \mathbb{R}$, satisfies $\sigma_{p}\left(C_{\varphi}\right)=\{1\}$ and $\sigma\left(C_{\varphi}\right)=\mathbb{C}$.

To mention a concrete explicit example, if $\delta(x)=e^{x}-e^{-x}$, then $\delta^{-1}(x)=\log \left(\frac{1}{2}\left(\left(y^{2}+4\right)^{1 / 2}-\right.\right.$ $y))$. 


\section{Quadratic function}

We now investigate the spectrum $\sigma\left(C_{\varphi}\right)$ of $C_{\varphi}: \mathscr{A}(\mathbb{R}) \rightarrow \mathscr{A}(\mathbb{R})$, when $\varphi(x)=a x^{2}+b x+c, a \neq 0$. We distinguish several cases, depending on the number of fixed points on $\varphi$. Lemma 3.13 will be used to reduce the problem to the study of certain parameter family.

Case 1. The symbol $\varphi$ has no fixed points. In this case, since $\varphi$ has only one critical point, it follows from Corollary 3.1 that $\sigma\left(C_{\varphi}\right)=\mathbb{C}$ and $\sigma_{p}\left(C_{\varphi}\right)=\mathbb{C} \backslash\{0\}$.

Case 2 and 3. The symbol has fixed points $v, u$ (possibly $u=v$ ) and

$$
\varphi(x)=x+a(x-u)(x-v), \quad a \neq 0 .
$$

Choose $u \leq v$ if $a<0$ or $u>v$ if $a>0$. then taking

$$
\delta(x)=-\frac{1}{a} x+u
$$

we will obtain

$$
\psi(x)=\delta^{-1} \circ \varphi \circ \delta(x)=[1+a(u-v)] x-x^{2} .
$$

Clearly, $u, v$ were selected to ensure that $\mu:=1+a(u-v) \geq 1$. By Lemma 3.13, it is enough to consider

$$
\varphi(x)=-x^{2}+\mu x \quad \text { with } \mu \geq 1 .
$$

Case 2. The symbol $\varphi$ has only one fixed point $u$. Then the behaviour of $C_{\varphi}$ is the same as $C_{\psi}$ for $\psi(x)=-x^{2}+x$.

Proposition 4.1 In Case 2, $\varphi(x)=-x^{2}+x, \sigma_{p}\left(C_{\varphi}\right)=\{1\}$ but $\sigma\left(C_{\varphi}\right) \supseteq\{0\} \cup[1,+\infty)$.

Proof: Clearly, by Theorem 2.2 and Proposition 2.1 we have

$$
\sigma_{p}\left(C_{\varphi}\right)=\{1\}, \quad \sigma\left(C_{\varphi}\right) \supset\{1,0\} .
$$

Now, we consider the map $T: \mathscr{A}(\mathbb{R}) \rightarrow \mathscr{A}(\mathbb{R}), T(f)=C_{\varphi}(f)-\lambda f$, and we will show that the function id, id $(x)=x$, does not belong to the image of $T$ for $\lambda \in(1,+\infty)$.

It is a simple calculation that there is the unique formal solution $f(z)=\sum_{n=0}^{\infty} f_{n} z^{n}$ of the equation $T(f)=$ id, with

$$
f_{0}=0, \quad f_{1}=\frac{1}{1-\lambda}, \quad f_{n}=\frac{1}{1-\lambda} \sum_{j=1}^{\left[\frac{n}{2}\right]}\left(\begin{array}{c}
n-j \\
j
\end{array}\right)(-1)^{j-1} f_{n-j} .
$$

It is clear that for $1-\lambda<0, f_{1}<0$ and $f_{2}>0$. Then one shows inductively by (5) that $f_{n}(-1)^{n-1}>0$. Therefore

$$
\left|f_{n}\right|>\frac{1}{|1-\lambda|}\left(\begin{array}{c}
n-1 \\
1
\end{array}\right)\left|f_{n-1}\right|=\frac{n-1}{|1-\lambda|}\left|f_{n-1}\right|
$$

Again inductively

$$
\left|f_{n}\right|>\frac{(n-1) !}{|1-\lambda|^{n}}
$$

and the series $\sum_{n=0}^{\infty} f_{n} z^{n}$ is nowhere convergent. So there is no real analytic solution $f$ for the equation $T(f)=$ id for any $\lambda>1$.

In Case 2. we also get the following partial positive step. 
Proposition 4.2 Let $\varphi(x)=-x^{2}+x, \lambda \neq 0$, and assume that the equation

$$
f(\varphi(x))-\lambda f(x)=\gamma(x)
$$

has an analytic solution $f$ on $(-\varepsilon, \varepsilon)$ for some $\varepsilon>0$ and some fixed $\gamma \in \mathscr{A}(\mathbb{R})$. Then this solution extends to a solution on $\mathbb{R}$.

Remark 4.3 Note that (6) has always the unique formal solution - we do not know for precisely which $\lambda$ and $\gamma$ it converges around zero.

Proof: We observe that for $x \in(0,1) \varphi^{[n]}(x) \rightarrow 0$ as $n \rightarrow \infty$ so, by Lemma 3.3, we extend $f$ to $(-\varepsilon, 1)$.

Since $\varphi:(-\infty, 1 / 2) \rightarrow(-\infty, 1 / 4)$ is a diffeomorphism onto, its inverse $\psi$ is a diffeomorphism $(-\infty, 1 / 4) \mapsto(-\infty, 1 / 2)$. Moreover, for $x \in(-\infty, 1 / 2)$ the equation

$$
f(\varphi(x))-\lambda f(x)=\gamma(x)
$$

is equivalent to

$$
f(\psi(x))-(1 / \lambda) f(x)=-(1 / \lambda) \gamma(\psi(x)) .
$$

Since for $x \in(-\infty, 0) \psi^{[n]}(x) \rightarrow 0$ as $n \rightarrow \infty$ we can extend $f$ onto $(-\infty, 0)$ by Lemma 3.3.

Summarizing, $f$ is a solution of $(6)$ on $(-\infty, 1)$.

Since for $x \in(0,1 / 2)$ and $y=1-x \in(1 / 2,1)$ we have

$$
\lambda f(x)+\gamma(x)=f(\varphi(x))=f(\varphi(y))=\lambda f(y)+\gamma(y)
$$

we have

$$
f(y)=f(1-y)+\frac{\gamma(1-y)-\gamma(y)}{\lambda} .
$$

This formula extends $f$ analytically onto $(1 / 2,+\infty)$ and so on the whole real line. Clearly, this extension satisfies (6) everywhere.

Case 3. The symbol $\varphi$ has exactly two different fixed points $u, v$. Then the behaviour of $C_{\varphi}$ is the same as $C_{\psi}$ for

$$
\psi(x)=-x^{2}+\mu x, \quad \mu>1 .
$$

The map $\psi$ has one critical point $\mu / 2$ and fixed points 0 and $\mu-1$.

Case 3.1. Critical point is outside the interval joining fixed points, i.e., $\mu / 2>\mu-1$ so $1<\mu<2$

Case 3.2. Critical point is equal to one fixed point, i.e., $\mu / 2=\mu-1$ so $\mu=2$.

Case 3.3. Critical point is between fixed points, i.e., $\mu / 2<\mu-1$ so $\mu>2$.

Proposition 4.4 In Case 3.1 and 3.2, $\varphi(x)=-x^{2}+\mu x, 1<\mu \leq 2, \sigma\left(C_{\varphi}\right)=\mathbb{C}$ but $\sigma_{p}\left(C_{\varphi}\right)=$ $\{1\}$ and for every $\lambda \in \mathbb{C}$ kernel of $C_{\varphi}-\lambda I$ is one-dimensional.

Proof: We define

$$
U_{1}:=(-\infty, \mu-1) \cup(1,+\infty), \quad U_{0}:=(0, \mu) .
$$

Observe that for every $x \in(-\infty, \mu-1)$ there is exactly one point $y \in(1, \infty)$ such that $\varphi(x)=$ $\varphi(y)(y=\mu-x)$. Thus $f \in \mathscr{A}\left(U_{1}\right)$ belongs to the kernel of $C_{\varphi}-\lambda I, \lambda \neq 0$ if and only if $\left.f\right|_{(-\infty, \mu-1)} \in \operatorname{ker}\left(C_{\varphi}-\lambda I\right)$ on $\mathscr{A}(-\infty, \mu-1)$ and

$$
f(y)=f(\mu-y), \quad y \in(1,+\infty) .
$$


Therefore the kernels of $C_{\varphi}-\lambda I$ on $\mathscr{A}\left(U_{1}\right)$ and on $\mathscr{A}(-\infty, \mu-1)$ have the same dimension. In the latter case $\varphi$ and $\varphi^{[2]}$ have only one fixed point 0 on $(-\infty, \mu-1)$. Hence, by Theorem 2.2, $\operatorname{ker} C_{\varphi}-\lambda I$ is one dimensional in $\mathscr{A}\left(U_{1}\right)$.

Since $\varphi:(0, \mu) \rightarrow(0, \mu)$ and its square have exactly one fixed point $\mu-1, \operatorname{ker} C_{\varphi}-\lambda I$ is one dimensional in $\mathscr{A}\left(U_{0}\right)$ by Theorem 2.2 .

Finally, $U_{1} \cap U_{0}=(0, \mu-1) \cup(1, \mu)$. Again as in case of $U_{1}$ one can prove that the kernels of $C_{\varphi}-\lambda I$ in $\mathscr{A}\left(U_{1} \cap U_{0}\right)$ and in $\mathscr{A}(0, \mu-1)$ are isomorphic. Since $\varphi$ has no fixed point in $(0, \mu-1)$ and only one critical point, the required kernel is infinite dimensional by Theorem 2.2 .

We apply Theorem 3.11 to conclude that, for every $\lambda \neq 0$, the map $C_{\varphi}-\lambda I: \mathscr{A}(\mathbb{R}) \rightarrow \mathscr{A}(\mathbb{R})$ is not surjective. By Proposition 2.1, $\sigma\left(C_{\varphi}\right)=\mathbb{C}$.

The remaining parts of the Proposition follows from Theorem 2.2.

In Case 3.3 we have only the following partial result:

Proposition 4.5 If $\varphi(x)=-x^{2}+\mu x, \mu>2$, then $\sigma\left(C_{\varphi}\right) \supset\{\lambda \in \mathbb{C}:|\lambda| \leq 1\} \cup\left\{\mu^{n}\right\}_{n} \cup\{(2-$ $\left.\mu)^{n}\right\}_{n}$.

Proof: Clearly, $\{0,1\} \cup\left\{\mu^{n}\right\}_{n} \cup\left\{(2-\mu)^{n}\right\}_{n} \subset \sigma\left(C_{\varphi}\right)$ by Propositions 2.1 and 2.2.

Fix $\lambda \neq 1,0<|\lambda| \leq 1$. Since $\varphi(1)=\mu-1$, we can select a sequence $x_{n} \in(0,1) \subset(0, \mu)$, $x_{1}=1$, such that

$$
\varphi^{[n]}\left(x_{n}\right)=\mu-1, \quad n \in \mathbb{N}
$$

Such $x_{n}$ exists because $\varphi(\mu / 2)=\mu^{2} / 4 \geq \mu$ and $1<\mu / 2$. In fact, $x_{n}=\frac{\mu-\sqrt{\mu^{2}-4 x_{n-1}}}{2}=$ $\frac{2 x_{n-1}}{\mu+\sqrt{\mu^{2}-4 x_{n-1}}}, n \in \mathbb{N}$. Hence $x_{n} / x_{n-1}<2 / \mu<1, n \in \mathbb{N}$, and $\left(x_{n}\right)_{n \in \mathbb{N}}$ is decreasing to zero as $n \rightarrow \infty$. Moreover, $x_{n} / x_{n-1}$ tends to $1 / \mu$ as $n \rightarrow \infty$.

If $C_{\varphi} f-\lambda f=\gamma$ for some $\gamma \in \mathscr{A}(\mathbb{R})$, then

$$
\lambda f(\mu-1)+\gamma(\mu-1)=f(\mu-1), \quad \text { so } \quad f(\mu-1)=\gamma(\mu-1) /(1-\lambda),
$$

and analogously

$$
f(0)=\frac{\gamma(0)}{1-\lambda},
$$

because both 0 and $\mu-1$ are fixed points for $\varphi$. Since

$$
f \circ \varphi^{[n]}(x)=\lambda^{n} f(x)+\lambda^{n-1} \gamma(x)+\lambda^{n-2} \gamma(\varphi(x))+\cdots+\gamma\left(\varphi^{[n-1]}(x)\right),
$$

we have

$$
\frac{\gamma(\mu-1)}{1-\lambda}=\lambda^{n} f\left(x_{n}\right)+\lambda^{n-1} \gamma\left(x_{n}\right)+\cdots+\lambda \gamma\left(\varphi^{[n-2]}\left(x_{n}\right)\right)+\gamma(1) .
$$

Fix $c<1 / 6$. If $\gamma(0)=0$, we can choose $n_{1}>2$ so that, for $n \geq n_{1},\left|x_{n}\right| \leq c$ and $\left|f\left(x_{n}\right)\right| \leq c$, since $f\left(x_{n}\right)$ tends to $f(0)=\gamma(0) /(1-\lambda)=0$.

Take $\gamma(x)=x^{k} \gamma_{0}(x)$, with $\gamma_{0} \in \mathscr{A}(\mathbb{R})$ satisfying $\gamma_{0}(\mu-1)=0$ and $1 / 2<\left|\gamma_{0}(x)\right|<1$ for $x \in[0,1), \gamma_{0}(1)=1$. Since $x_{n} / x_{n-1}$ tends to $1 / \mu$ as $n \rightarrow \infty$, there is $n_{0}>n_{1}$ such that $x_{n}<(1 / 2) x_{n-1}$ for $n>n_{0}$. We can take $k$ big enough to ensure that $\left|\gamma\left(x_{j}\right)\right|<c / n_{0}$ for $2 \leq j \leq n_{0}$. Then, for $n>n_{0}$, we have $\left|\gamma\left(x_{n}\right)\right| \leq 2 c(1 / 2)^{n-n_{0}}$. Summarizing:

$$
\left|\lambda^{n} f\left(x_{n}\right)+\lambda^{n-1} \gamma\left(x_{n}\right)+\cdots+\lambda \gamma\left(\varphi^{[n-2]}\left(x_{n}\right)\right)\right| \leq 2 c+2 c \frac{1}{1-(1 / 2)^{k}} \leq 6 c .
$$

As $c<1 / 6$, we reach a contradiction with (7). Therefore $C_{\varphi}-\lambda I$ is not surjective. 
Acknowledgement: The research of the authors was partially supported by MEC and FEDER Project MTM2013-43540-P and the work of of Bonet by the grant GV Project Prometeo II/2013/013. The research of Domański was supported by National Center of Science, Poland, grant no. DEC-2013/10/A/ST1/00091.

\section{References}

[1] G. Belitskii, Yu. Lyubich, The Abel equation and total solvability of linear functional equations, Studia Math. 127 (1998), 81-97.

[2] G. Belitskii, Yu. Lyubich, The real analytic solutions of the Abel functional equation, Studia Math. 134 (1999), 135-141.

[3] G. Belitskii, V. Tkachenko, One-Dimensional Functional Equations, Springer, Basel, 2003.

[4] G. Belitskii, V. Tkachenko, Functional equations in real analytic functions, Studia Math. 143 (2000), 153-174

[5] J. Bonet, P. Domański, Power bounded composition operators on spaces of analytic functions, Collect. Math. 62 (2011), 69-83.

[6] J. Bonet, P. Domański, Hypercyclic composition operators on spaces of real analytic fucntions, Math. Proc. Cambridge Phil. Soc. 153 (2012), 489-503.

[7] J. Bonet, P. Domański, Abel's functional equation and eigenvalues of composition operators on spaces of real analytic functions, Integral Equations Operator Theory 81 (2015), 455-482, DOI 10.1007/s00020-014-2175-4.

[8] H. Cartan, Variétés analytiques réelles et variétés analytiques complexes, Bull. Soc. Math. France 85 (1957), 77-99.

[9] P. Domański, Notes on real analytic functions and classical operators, Topics in Complex Analysis and Operator Theory (Winter School in Complex Analysis and Operator Theory, Valencia, February 2010), Contemporary Math. 561 (2012) 3-47. Amer. Math. Soc., Providence, 2012.

[10] P. Domański, M. Goliński and M. Langenbruch, A note on composition operators on spaces of real analytic functions, Ann. Polon. Mat. 103 (2012), 209-216.

[11] P. Domański, M. Langenbruch, Composition operators on spaces of real analytic functions, Math. Nachr. 254-255 (2003), 68-86.

[12] P. Domański, M. Langenbruch, Coherent analytic sets and composition of real analytic functions, J. reine angew. Math. $\mathbf{5 8 2}$ (2005), 41-59.

[13] P. Domański, M. Langenbruch, Composition operators with closed image on spaces of real analytic functions, Bull. London Math. Soc. 38 (2006), 636-646.

[14] P. Domański, D. Vogt, The space of real analytic functions has no basis, Studia Math. 142 (2000), 187-200.

[15] L. Hörmander, An Introduction to Complex Analysis in Several Variables, North Holland, Amsterdam, 1986. 
[16] R. Meise, D. Vogt, Introduction to Functional Analysis, Clarendon Press, Oxford 1997.

[17] W. Smajdor, On the existence and uniqueness of analytic solutions of the functional equation $\varphi(z)=h(z, \varphi[f(z)])$, Ann. Polon. Math. 19 (1967), 37-45. 